Manuscripts, books for review, and other communications for the English-language editor should be sent to John Woods, Department of Philosophy, University of Lethbridge, Lethbridge, Alberta, T IK $2 \mathrm{M} 4$.

Articles, notes, livres pour comptes rendus et comptes rendus rédigés en français devront parvenir à François Duchesneau, Faculté de Philosophie, Université d'Ottawa, Ottawa, Ontario, K IN 6N5.

All advertising copy should be addressed to Joanne S. McGlynn, Administrative Secretary, Canadian Philosophical Association.

Pour les annonces à insérer dans la revue, prière de s'adresser à Joanne A. McGlynn, secrétaire administratif, Association canadienne de Philosophie.

Membership/Cotisation annuelle: \$25.00; Student/Étudiants: \$8.00; Subscriptions/Abonnements: $\$ 30.00$; Single Copies/Le numéro: $\$ 8.00$.

Cheques should be made payable to the Canadian Philosophical Association. Tout paiement doit être établi à l'ordre de l'Association canadienne de Philosophie.

Association canadienne de Philosophie Suite 46, I 390 ouest, rue Sherbrooke Montréal, Quebec

H3G IK2
Canadian Philosophical Association Suite 46, 390 Sherbrooke Street West Montreal, Quebec, H3G IK2 


\section{Collection RECHERCHIES}

\section{SECTION PHILOSOPHIE}

BENOIT PRUCHE

EXISTANT ET ACTE D'ETRE. Essai de philosophie existentielle Tome I :

Introduction générale - Critique existentielle

Cet essai a pour but de dégager les cheminements d'une métaphysique à partir d'une phénoménologie de la conscience de soi. Après une Introduction destinée à mettre le lecteur dans le plan existentiel défini par l'auteur, la partie CRITIQUE de cette recherche porte sur la valeur de la connaissance. Inspirée du cheminement phénoménologique. la méthode fait droit aux données expérimentales d'èvidence immédiate provenant d'une analyse très poussée de la subjectivité comme conscience de soi. Une deuxième partie. ANALYTIQUE, dégagera une structure métaphysique.

212 pages

PITRRL LUCIER

\section{EMPIRISME LOGIQUE ET LANGAGE RELIGIEUX}

Trois approches anglo-saxonnes contemporaines: R.B. Braithwaite. R.M. Hare, 1.T. Ramsey

L intérèt pour l'analyse du langage religieux est devenu un phénomène culturel massif. C'est aux problèmes posés par ce langage, dans l'univers des dires de l'homme, que s'attaque le présent ouvrage. A travers et par-delà l'analyse de trois (euvres anglo-saxonnes contemporaines, l'autcur fait émerger, interprète et critique une problématique. II pose ainsi les jalons de ce que pourrait ètre une approche praxéologique du langage religieux.

462 pages

Parus priciedemment

dans la même collection

L.AURE.NT GIROUX

DUREE PURE ET TEMPORALITE. BERGSON ET HEIDEGGER

136 pages

JULII:N NAUD

STRUCTURE ET SENS DU SYMBOLE. L IMAGINAIRE CIIT. (IASION BACHELARD

232 pages

\section{LES EDITIONS BELLARMIN 8I00, BOULEVARD SAINT-LAURENT MONTRÉAL H2P 2 L9$$
\text { TÉL.: (514) 387-254I }
$$ 